\title{
PREPROCESSING AND REGISTRATION OF MINISCOPE-BASED CALCIUM IMAGING OF THE RODENT BRAIN
}

\author{
Tamerlan B. Sagindykov ${ }^{1}$, Alexey R. Brazhe ${ }^{2}$, Dmitry V. Sorokin ${ }^{1, *}$ \\ ${ }^{1}$ Laboratory of Mathematical Methods of Image Processing, Faculty of Computational Mathematics and Cybernetics, \\ Lomonosov Moscow State University, Moscow, Russia - dsorokin@cs.msu.ru \\ ${ }^{2}$ Department of Biophysics, Faculty of Biology, \\ Lomonosov Moscow State University, Moscow, Russia - brazhe@gmail.com
}

Commission II, WG II/10

KEY WORDS: Image registration, neurons, live tissue imaging, miniscope

\begin{abstract}
:
Microscopic imaging is central to the brain and cognition studies in animals and often requires advanced image processing. In vivo recordings on awake behaving animals require stabilization of the images as the tissue in the images undergoes non-rigid deformations due to animal movement, pulse beat and breathing of the animal. Here we propose an approach to compensation for the tissue motion in calcium imaging data acquired with miniaturized wearable microscopes (miniscopes) from live rodent brains. Our approach includes preprocessing of the images in which we compensate for non-uniform illumination, remove calcium transients and instrument-specific noise. For image registration we use the multiscale mutual information based non-rigid algorithm with B-spline transformation model. We present the preliminary results of such motion compensation approach applied to the real miniscope image stacks.
\end{abstract}

\section{INTRODUCTION}

Brain studies heavily rely on imaging applications, placing image processing tools in high demand (Hamel et al., 2015). For example, fluorescent calcium imaging allows to visualize activity of large groups of neurons or astrocytes. A currently popular technique is to use a combination of genetically encoded calcium reporters and wearable miniaturized micro-endoscopes (miniscopes). This allows imaging with cellular resolution in freely behaving animals, with fluctuations of image intensity over time providing a read-out of undergoing activity of the cells, expressing the calcium reporter protein (Fig. 1a).

Reliable analysis of calcium activity in the neural tissue implies stationary sources of activity. However, in vivo recordings in awake and moving animals are particularly prone to movement artifacts - distortions of the brain tissue relative to the GRIN lens and the light sensor of the miniscope — despite the best efforts of clamping the miniscope to the skull of the animal. Thus, the tissue in the recorded image sequence undergoes a nonrigid motion caused by heart beat, breathing and head movement. This motion can be compensated by means of image registration, namely by aligning all the imaged frames to a stationary template, usually the first image in a series.

Previously, several cell image registration methods have been presented. In (Ozere et al., 2013) the authors suggested a parametric motion model for cell motion compensation. However, the approach can compensate only affine changes. In (Kim et al., 2011, Sorokin et al., 2018) the non-rigid cell nuclei registration methods were proposed. The aim of the algorithm was to compensate for the cell global motion in order to analize solely the local motion of subnuclear particles. The registration method proposed in (Thevenaz et al., 1998) employed multiscale splinebased approach. In the keypoint-based approach proposed in (Ka-

\footnotetext{
${ }^{*}$ Corresponding author
}

jihara et al., 2017) the authors perform non-rigid registration by combination of affine registration of image parts each performed using a cluster of keypoints. In (Lu et al., 2010) the authors use the global-to-local approach, where the global rigid transform is performed using the contours of the object, and the local transformation is obtained using th SIFT descriptors (Lowe, 1999). One of the most common approaches to non-rigid intensity-based registration is to pose image registration as an optimization problem (Klein et al., 2010). In this case, one has to choose a parametrized coordinate transformation model to be optimized, a cost function, an optimization method, and image interpolation method. Such approaches are usually applied iteratively. A multiscale strategy (Lester and Arridge, 1999) can be also used to improve the registration results. In (Klein et al., 2010) the authors present Elastix framework that has modular design and allows to choose from different transformation models, cost functions, optimization and interpolation methods.

In this work, we aim at compensating for the live rodent brain tissue motion in image sequences by means of image registration. The motion of the animal, slight brain swelling, and changes in local blood flow lead to the elastic transformation of the brain tissue. The registration of live rodent brain image sequences is challenging due to the non-uniform illumination of the image sequence in space and time and low signal-to-noise ratio. Additionally, fluorescence intensity changes with calcium activity, which makes it impossible to rely on constant brightness models. First, we preprocess the images to remove the experimentspecific noise caused by dust on the optics and CCD sensor. Next, we perform non-uniform illumination correction, and gammacorrection of the images. We also suppress the calcium activity affecting the resulting deformation field as the algorithm attempts to register also the calcium transients in addition to the motion of the brain tissue (Fig. 1b). Finally, we perform the image registration using the multiscale B-spline approach with random coordinate sampler, advanced Mattes mutual information as the cost 
function, and stochastic gradient descent as the optimizer. We used the SimpleElastix library to perform the image registration (Marstal et al., 2016). We applied our approach for the real image sequence of the live rodent brain. The preliminary qualitative and quantitative results look promising.

\section{METHODS}

In this section, we describe the preprocessing and registration approaches that we used to compensate for the tissue motion.

\subsection{Preprocessing}

As the first step of preprocessing we remove the data-specific noise from each image of the rodent brain tissue image sequence $\left\{I_{n}(\mathbf{x})\right\}$. This noise is probably associated with dust and other artifacts on the CCD matrix and optics. To this end, we calculate the residue image

$$
r_{n}(\mathbf{x})=I_{n}(\mathbf{x})-F_{\text {median }}\left[I_{n}(\mathbf{x}), 5\right]
$$

where $n$ is the image index, and $F_{\text {median }}[., r]$ is the median filter with radius $r$. The average residue image corresponds to the nonmoving structures like dust, so we subtract it from each image of the sequence to remove such noise

$$
\bar{I}(\mathbf{x})=I_{n}(\mathbf{x})-\frac{1}{N} \sum_{n} r_{n}(\mathbf{x}) .
$$

Next, we perform the illumination correction. To obtain the illumination patterns of the image on different scales we blur it with a bank of Gaussian filters with different $\sigma$ (Jobson et al., 1997). Then the image with the corrected illumination is computed as

$$
\overline{\bar{I}}(\mathbf{x})=\frac{1}{N_{\sigma}} \sum_{i=1}^{N_{\sigma}} \log \frac{\bar{I}(\mathbf{x})}{G_{\sigma_{i}}[\bar{I}(\mathbf{x})]},
$$

where $G_{\sigma_{i}}[$.$] is the Gaussian filter with sigma \sigma_{i}$, and $N_{\sigma}$ is the number of scales. Applying it to each image of the sequence we suppress the non-uniform illumination, however the average intensity can still vary between the frames. To correct it, we modify the intensity in every frame as

$$
\widetilde{I_{n}}(\mathbf{x})=\left(\overline{\overline{I_{n}}}(\mathbf{x})-m_{n}\right) \frac{s_{1}}{s_{n}}+m_{1},
$$

where $n$ is the frame number, $m_{n}$ is the mean of the $n$-th frame intensity, and $s_{n}$ is the standard deviation of the $n$-th frame intensity. Finally, we perform gamma-correction for every frame with $\gamma=0.65$.

The last step of the image preprocessing is the suppression of calcium activity as the changes of intensities across the images caused by the calcium activity significantly decrease the image registration performance. For this, we calculate the median value over time for each pixel of the image and replace pixel intensity with its median if its value is greater then the median:

$$
\hat{I_{n}}(\mathbf{x})= \begin{cases}\widetilde{I_{n}}(\mathbf{x}), & \widetilde{I_{n}}(\mathbf{x}) \leq I_{\text {median }}(\mathbf{x}) \\ I_{\text {median }}(\mathbf{x}), & \text { otherwise }\end{cases}
$$

where $I_{\text {median }}(\mathbf{x})$ is the median over time image. The results of the preprocessing are presented in Fig. $1 \mathrm{~b}$.

\subsection{Registration}

The compensation for the tissue motion in the image sequence is performed by means of pairwise image registration. The goal is to obtain a non-rigid coordinate transform $\mathbf{T}_{n}(\mathbf{x})$ for each pair of consequent frames $I_{n}(\mathbf{x})$ and $I_{n+1}(\mathbf{x})$ that registers the image $I_{n+1}(\mathbf{x})$ to $I_{n}(\mathbf{x})$. We perform the registration for the preprocessed images $\hat{I}(\mathbf{x})$ and consequently apply the obtained transformations to register the initial image $I_{n}(\mathbf{x})$ to the first image of the sequence $I_{1}(\mathbf{x})$. To obtain the transformations $\mathbf{T}_{n}(\mathbf{x})$ we use elastix framework (Klein et al., 2010). First, we compensate for the affine part of the transformation. Then the non-rigid part is obtained using the multiscale B-spline approach with 4 scales and a random coordinate sampler. We use advanced Mattes mutual information with bending energy penalty as the registration cost function and stochastic gradient descent as the optimizer.

Below we describe the registration process in detail. Two images are involved: let us denote the fixed template image as $I_{F}(\mathbf{x})$ and the moving image as $I_{M}(\mathbf{x})$. The moving image is deformed to fit the template. The quality of alignment is defined by the distance in the mutual information $(M I)$ metric. Because the problem is ill-posed for nonrigid transformations $\mathbf{T}$, a regularisation term $P$ that constrains $\mathbf{T}$ is often introduced. The registration problem is formulated as an optimisation problem in which the cost function $C$ is minimised:

$$
\begin{aligned}
\mathbf{T}_{r e g} & =\arg \min _{\mathbf{T}} C\left(\mathbf{T} ; I_{F}, I_{M}\right), \\
C\left(\mathbf{T} ; I_{F}, I_{M}\right) & =M I\left(\mathbf{T} ; I_{F}, I_{M}\right)+\alpha P(\mathbf{T}),
\end{aligned}
$$

where $\alpha$ is the regularization parameter. If the minimization problem is solved for a parametrized transform $\mathbf{T}=\mathbf{T}_{\boldsymbol{\mu}}$ the equation (2) is formed as

$$
\boldsymbol{\mu}_{\text {reg }}=\arg \min _{\boldsymbol{\mu}} C\left(\mathbf{T}_{\boldsymbol{\mu}} ; I_{F}, I_{M}\right),
$$

where $\boldsymbol{\mu}$ is a vector of transform parameters.

In this work, we use the combination of affine and B-spline transforms for $\mathbf{T}_{\mu}$ :

$$
\begin{aligned}
\mathbf{T}_{\boldsymbol{\mu}} & =\mathbf{A}_{\boldsymbol{\mu}}+\mathbf{B}_{\boldsymbol{\mu}} \\
\mathbf{A}_{\boldsymbol{\mu}_{A}}(\mathbf{x}) & =\mathbf{M}(\mathbf{x}-\mathbf{c})+\mathbf{t}+\mathbf{c} \\
\mathbf{B}_{\boldsymbol{\mu}_{B}}(\mathbf{x}) & =\mathbf{x}+\sum_{\mathbf{x}_{k} \in N_{\mathbf{x}}} \mathbf{p}_{k} \beta^{3}\left(\frac{\mathbf{x}-\mathbf{x}_{k}}{\sigma}\right)
\end{aligned}
$$

where $\mathbf{A}_{\boldsymbol{\mu}_{A}}$ is the affine transform with matrix $\mathbf{M}, \mathbf{c}$ is the centre of rotation, and $\mathbf{t}$ is the translation vector. The second term $B_{\boldsymbol{\mu}}$ is the B-spline transform with the control points $\mathbf{x}_{k}$ defined on a regular grid, $\beta^{3}(\mathbf{x})$ is the cubic multidimensional $\mathrm{B}$-spline polynomial, $\mathbf{p}_{k}$ are the B-spline coefficient vectors, $\sigma$ is the B-spline control point spacing, and $N_{\mathrm{x}}$ the set of all control points within the compact support of the B-spline at $\mathbf{x}$. Thus, the parameter vector $\boldsymbol{\mu}$ consists of affine parameters $\boldsymbol{\mu}_{A}$ and B-spline transform parameters $\boldsymbol{\mu}_{B}$. The affine parameter vector $\boldsymbol{\mu}_{A}$ contains matrix $\mathbf{M}$ elements and translation vector $\mathbf{t}$. The $\mathrm{B}$-spline parameter vector $\mu_{B}$ contains the coefficients $\left\{\mathbf{p}_{k}\right\}$.

The mutual Information (Mattes et al., 2003, Thévenaz and Unser, 2000) is defined as

$$
\begin{aligned}
M I\left(\mathbf{T}_{\boldsymbol{\mu}} ; I_{F}, I_{M}\right)=\sum_{m \in L_{M}} \sum_{f \in L_{F}} p(f, m ; \boldsymbol{\mu}) \times \\
\times \log _{2}\left(\frac{p(f, m ; \boldsymbol{\mu})}{p_{F}(f) p_{M}(m ; \boldsymbol{\mu})}\right),
\end{aligned}
$$


where $L_{F}$ and $L_{M}$ are sets of regularly spaced intensity bin centres, $p$ is the discrete joint probability, and $p_{F}$ and $p_{M}$ are the marginal discrete probabilities of the fixed and moving image, obtained by summing $p$ over $m$ and $f$, respectively. The joint probabilities are estimated using B-spline Parzen windows:

$$
\begin{aligned}
p(f, m ; \boldsymbol{\mu})=\frac{1}{\left|\Omega_{F}\right|} \sum_{\mathbf{x}_{i} \in \Omega_{F}} \omega_{F} & \left(\frac{f-I_{F}\left(\mathbf{x}_{i}\right)}{\sigma_{F}}\right) \times \\
& \times \omega_{M}\left(\frac{m-I_{M}\left(\mathbf{T}_{\boldsymbol{\mu}}\left(\mathbf{x}_{i}\right)\right)}{\sigma_{M}}\right),
\end{aligned}
$$

where $\mathbf{x}_{i}$ are the points of the the fixed image domain $\Omega_{F}, \omega_{F}$ and $\omega_{M}$ represent the fixed and moving B-spline Parzen windows. The scaling constants $\sigma_{F}$ and $\sigma_{M}$ must equal the intensity bin widths defined by $L_{F}$ and $L_{M}$. These follow directly from the grey-value ranges of $I_{F}$ and $I_{M}$ and the user-specified number of histogram bins $\left|L_{F}\right|$ and $\left|L_{M}\right|$.

For regularization term $P\left(\mathbf{T}_{\boldsymbol{\mu}}\right)$ in (2) we used the bending energy penalty (Rueckert et al., 1999):

$$
\begin{aligned}
P\left(\mathbf{T}_{\boldsymbol{\mu}}\right) & =\frac{1}{K} \sum_{\mathbf{x}_{i}}\left\|\frac{\partial^{2} \mathbf{T}_{\boldsymbol{\mu}}}{\partial \mathbf{x} \partial \mathbf{x}^{T}}\left(\mathbf{x}_{i}\right)\right\|_{F}^{2}= \\
& =\frac{1}{K} \sum_{\mathbf{x}_{i}}\left(\sum_{j=1}^{2}\left(\frac{\partial^{2} T_{\boldsymbol{\mu}_{j}}}{\partial^{2} x_{1}}\left(\mathbf{x}_{i}\right)\right)^{2}+\right. \\
& \left.+2\left(\frac{\partial^{2} T_{\boldsymbol{\mu}_{j}}}{\partial x_{1} \partial x_{2}}\left(\mathbf{x}_{i}\right)\right)^{2}+\left(\frac{\partial^{2} T_{\boldsymbol{\mu}_{j}}}{\partial^{2} x_{2}}\left(\mathbf{x}_{i}\right)\right)^{2}\right),
\end{aligned}
$$

where $\mathbf{x}_{i}$ are the points where $P\left(\mathbf{T}_{\boldsymbol{\mu}}\right)$ is computed, and $K$ is the number of such points. In this work, we used a random subset of image points $\mathbf{x}_{i}$ which is updated every iteration. Such approach significantly reduces the computation time (Staring et al., 2007).

To solve the optimisation problem (2) to obtain the optimal transformation parameter vector $\boldsymbol{\mu}_{\text {reg }}$ we use an iterative optimisation strategy with gradient descent

$$
\boldsymbol{\mu}_{k+1}=\boldsymbol{\mu}_{k}-\left.\eta_{k} \frac{\partial C}{\partial \boldsymbol{\mu}}\right|_{\boldsymbol{\mu}=\boldsymbol{\mu}_{k}}, k=0,1,2, \ldots
$$

where $\eta_{k}$ is the gradient descent step size. The optimization process stops when the difference between the $C\left(\mathbf{T}_{\boldsymbol{\mu}} ; I_{F}, I_{M}\right)$ between the iterations reaches some predefined threshold or the number of iterations exceeds the predefined limit.

During the optimization the value of $I_{M}(\mathbf{T}(\mathbf{x}))$ needs to be computed not at the pixel positions. For this, we used bilinear interpolation method.

We used the multiscale version of the described approach when fixed and moving images were represented as Gaussian scalespace (Lester and Arridge, 1999) and the transformation $\mathbf{T}(\mathbf{x})$ was found iteratively from coarse to fine scales.

\section{RESULTS AND CONCLUSIONS}

We applied our motion compensation approach to miniscope imaging data of live rodent cerebellum, with Purkinje cells expressing a genetically encoded calcium indicator, kindly provided by Dr. Tycho Hoogland (Netherlands Institute for Neuroscience). We used five 2D fluorescence image sequences acquired in single channel consisting of 1000 images with the resolution of $752 \times 480$.
The results of the global motion compensation approach are presented in Fig. 1c. In addition, we calculated the difference between the first frame and the $n$-th frame of the initial and registered sequences (Fig. 1d,e). The difference images for the initial image sequence (Fig. 1d) contain bright and dark structures indicating significant motion in the sequence. In contrast, the difference images for the registered sequence (Fig. 1e) show that most of the tissue motion has been eliminated as only the bright structures representing the calcium signaling are retained.

To evaluate the registration performance quantitatively we calculated the mean square error (MSE) between the first $\hat{I}_{1}(\mathbf{x})$ and the $n$-th $\hat{I_{n}}(\mathbf{x})$ preprocessed images. To demonstrate the variation of the registration quality in time, the graph of the MSE as a function of frame number is shown in Fig. 2. It can be seen that the MSE curve becomes more flat after for the registered sequence which reflects the lack of motion in the sequence. The remaining level of MSE after the registration is the subject of noise in the images. The average over sequences MSE values for the unregistered and registered image sequences were 0.25 and 0.18 which demonstrates the $26 \%$ decrease of the error.

\section{CONCLUSIONS}

In this work we present a practical approach to correct tissue motion artifacts in the live rodent brain imaging data. We describe the key points of the the image preprocessing steps and the registration approach. The preliminary qualitative results show that the motion of the tissue is successfully eliminated retaining only the calcium signaling events which are the subject of the study. Further analysis of the algorithm, optimization of the registration parameters and quantitative evaluation of the results are intended for future work.

\section{ACKNOWLEDGEMENTS}

This work has been supported by the Russian Foundation for Basic Research Grant No. komfi-17-00-00407, and by the Russian Science Foundation grant 17-11-01279 for image processing. We thank Dr. Tycho Hoogland (Netherlands Institute for Neuroscience) for kindly providing the data.

\section{REFERENCES}

Hamel, E. J., Grewe, B. F., Parker, J. G. and Schnitzer, M. J., 2015. Cellular level brain imaging in behaving mammals: an engineering approach. Neuron 86(1), pp. 140-159.

Jobson, D. J., Rahman, Z.-u. and Woodell, G. A., 1997. A multiscale retinex for bridging the gap between color images and the human observation of scenes. IEEE Transactions on Image processing 6(7), pp. 965-976.

Kajihara, T., Funatomi, T., Kubo, H., Makishima, H., Yamada, S., Aoto, T. and Mukaigawa, Y., 2017. Feature-based non-rigid registration of serial section images by blending rigid transformations. In: 4th IAPR Asian Conference on Pattern Recognition (ACPR), pp. 858-863.

Kim, I.-H., Chen, Y.-C. M., Spector, D. L., Eils, R. and Rohr, K., 2011. Nonrigid registration of 2-d and 3-d dynamic cell nuclei images for improved classification of subcellular particle motion. IEEE Transactions on Image Processing 20(4), pp. 1011-1022.

Klein, S., Staring, M., Murphy, K., Viergever, M. A. and Pluim, J. P., 2010. Elastix: a toolbox for intensity-based medical image registration. IEEE Transactions on Medical Imaging 29(1), pp. 196-205. 

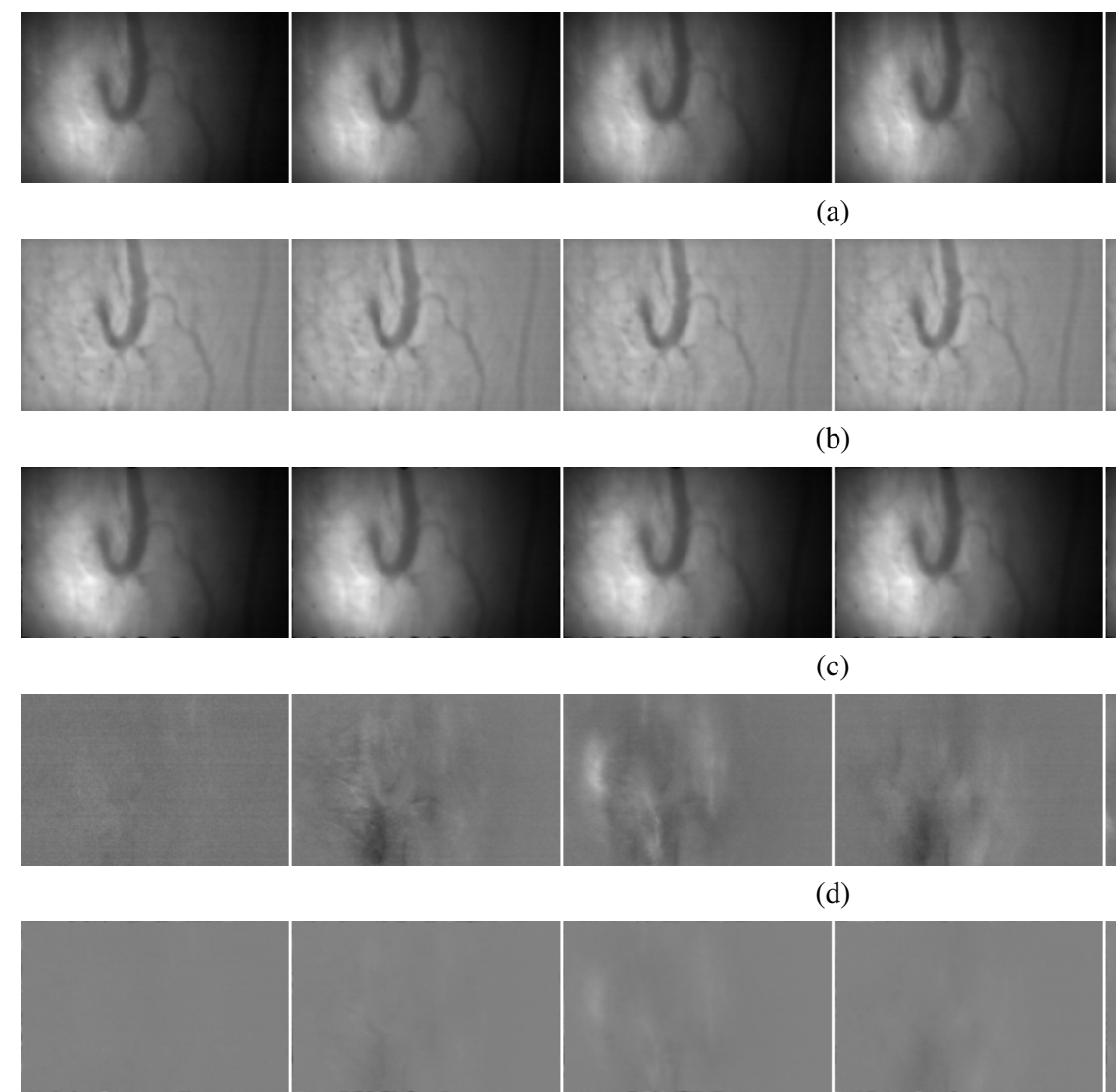

(e)

(a)

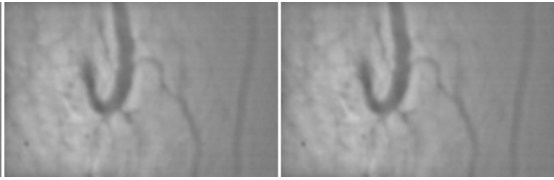

(b)

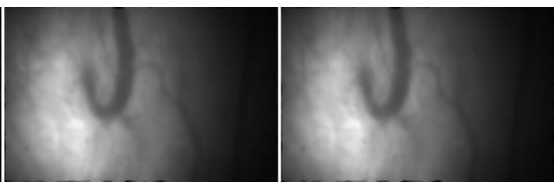

(c)

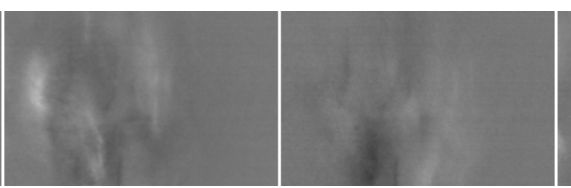

(d)
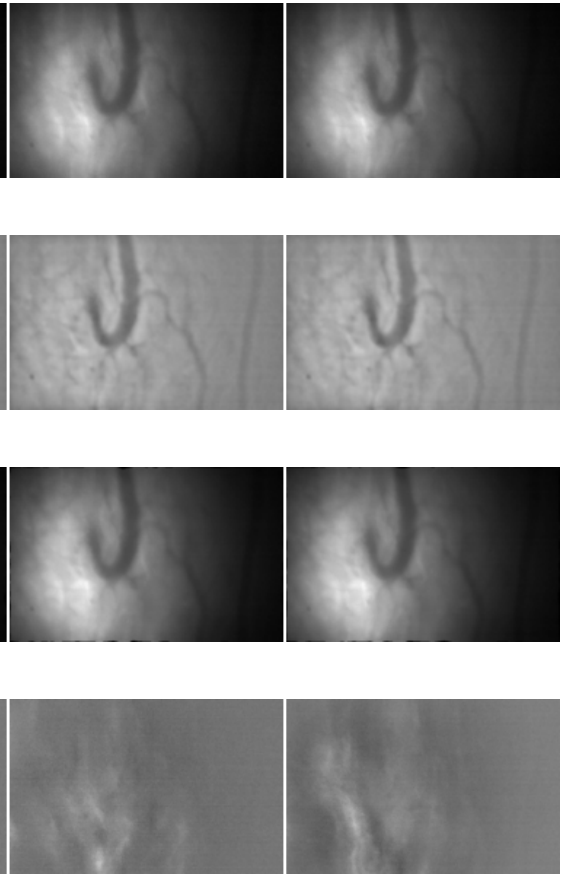

Figure 1. The every 165-th frame of the contrast-enhanced initial image sequence (a), the preprocessed image sequence (b), the registered image sequence (c), the difference between the first frame and $n$-th frame of the initial (d) and registered (e) sequences (contrast enhanced).

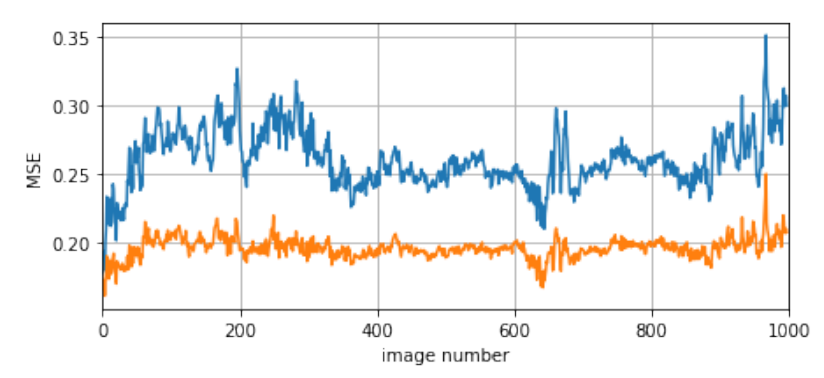

Figure 2. Mean square error between the first and $n$-th images of unregistered (blue line) and registered (orange line) sequences.

Lester, H. and Arridge, S. R., 1999. A survey of hierarchical non-linear medical image registration. Pattern Recognition 32(1), pp. 129-149.

Lowe, D. G., 1999. Object recognition from local scale-invariant features. In: The proceedings of the 7th IEEE International Conference on Computer Vision (ICCV), Vol. 2, pp. 1150-1157.

Lu, X., Zhang, S., Yang, W. and Chen, Y., 2010. Sift and shape information incorporated into fluid model for non-rigid registration of ultrasound images. Computer Methods and Programs in Biomedicine 100(2), pp. 123-131.

Marstal, K., Berendsen, F., Staring, M. and Klein, S., 2016. Simpleelastix: A user-friendly, multi-lingual library for medical image registration. In: Proc. of the IEEE Conference on Computer Vision and Pattern Recognition Workshops, pp. 134-142.
Mattes, D., Haynor, D. R., Vesselle, H., Lewellen, T. K. and Eubank, W., 2003. Pet-ct image registration in the chest using free-form deformations. IEEE Transactions on Medical Imaging 22(1), pp. 120-128.

Ozere, S., Bouthemy, P., Spindler, F., Paul-Gilloteaux, P. and Kervrann, C., 2013. Robust parametric stabilization of moving cells with intensity correction in light microscopy image sequences. In: The 10th IEEE International Symposium on Biomedical Imaging (ISBI 2013), pp. 468-471.

Rueckert, D., Sonoda, L. I., Hayes, C., Hill, D. L., Leach, M. O. and Hawkes, D. J., 1999. Nonrigid registration using free-form deformations: application to breast MR images. IEEE Transactions on Medical Imaging 18(8), pp. 712-721.

Sorokin, D. V., Peterlik, I., Tektonidis, M., Rohr, K. and Matula, P., 2018. Non-rigid contour-based registration of cell nuclei in 2d live cell microscopy images using a dynamic elasticity model. IEEE Transactions on Medical Imaging 37(1), pp. 173-184.

Staring, M., Klein, S. and Pluim, J. P., 2007. A rigidity penalty term for nonrigid registration. Medical Physics 34(11), pp. 40984108 .

Thévenaz, P. and Unser, M., 2000. Optimization of mutual information for multiresolution image registration. IEEE Transactions on Image Processing 9(12), pp. 2083-2099.

Thevenaz, P., Ruttimann, U. E. and Unser, M., 1998. A pyramid approach to subpixel registration based on intensity. IEEE Transactions on Image Processing 7(1), pp. 27-41.

Revised April 2019 